Investigaciones Fenomenológicas, n. 9, 2012, 11-38.

e-ISSN: $1885-1088$

\title{
EMPATÍA Y SER-PARA-OTRO. HUSSERL Y SARTRE \\ ANTE EL PROBLEMA DE LA INTERSUBJETIVIDAD
}

\author{
Pedro S. Alves \\ Universidade de Lisboa, Portugal \\ psalves2@gmail.com
}

\begin{abstract}
Resumen: En este documento, debato ampliamente sobre la fenomenología de la intersubjetividad presentada por Husserl y por Sartre, centrándome en los conceptos de empatía y ser-para-otros. Contrariamente a la opinión más común, rechazo la objeción de solipsista dirigida a Husserl y sostengo que es la descripción de Sartre la que da lugar a esa objeción, en la medida en que él describe la conciencia de otro sujeto como una forma de autoconocimiento como un "objeto" (para "otros"). Termino señalando algunas directrices para convertir el análisis de Husserl de la aprehensión de otros sujetos en una fenomenología de la comunicación del más alto nivel.
\end{abstract}

Palabras clave: Intersubjetividad, empatía, ser-para-los-otros, solipsismo, comunicación.

\begin{abstract}
In this paper, I discuss at length the phenomenology of intersubjectivity put forward by Husserl and Sartre, focusing on the concepts of empathy and being-for-others. Contrary to the main opinion, I refuse the solipsist objection addressed to Husserl, and I argue that it is Sartre's description that gives rise to such an objection, insofar as he describes the consciousness of another subject as a form of selfconsciousness as an "object" (for "other"). I finish pointing to some guidelines to convert Husserl's analysis of the apprehension of other subjects into a fully-fledged phenomenology of communication.
\end{abstract}

Palabras clave: Intersubjectivity, empathy, being-for-others, solipsism, communication.

\section{SOLIPSISMO Y FENOMENOLOGÍA TRANSCENDENTAL}

Hay un problema que ha sobrevolado como un espectro las teorías fenomenológicas de la intersubjetividad. Me refiero al problema del solipsismo. Desde que, en el primer párrafo de la quinta meditación cartesiana, Edmund Husserl dirigió un argumento solipsista contra su propia fenomenología trascendental, interpretada como un idealismo, ésta no ha cesado de tener que confrontarse con y explicarse ante esa objeción. En los términos de su formulación husserliana, la objeción solipsista está contenida por entero en una perplejidad que tiene su raíz en la propia posición reflexiva instauradora de la fenomenología:

Cuando yo, el yo que medita, me reduzco a mi ego trascendental absoluto mediante la epoché fenomenológica, ¿no me convierto en un solus ipse y no permanezco como tal 
en cuanto que yo, bajo el título Fenomenología, ejerzo consecuentemente una autoexplicitación? ¿No deberá una fenomenología, que quiere resolver los problemas del ser objetivo y presentarse como Filosofía, ser estigmatizada como solipsismo trascendental ${ }^{1}$

El problema del solipsismo es, aquí, inmediatamente presentado en su configuración más general: es la consecuencia, aparentemente irremediable, de una filosofía que reduce todo ser al para mí en que se constituye como objeto, mientras que a propósito del alter-ego, este no puede ser más un ser que no es más que "para mí", en cuanto objeto constituido en mi experiencia, sino que será para sí mismo un ser, y un ser que, independientemente de su devenir objeto para otra conciencia, habla para sí mismo y se experimenta a sí mismo como un "yo". ¿Cómo es posible una doctrina de la constitución del alter-ego como un ser para sí, si constituir significa reenviar ese "para sí" al para mí de mi experiencia? Tal es el desafío lanzado por una paradójica "constitución" de la alteridad, tal es la amenaza permanente de solipsismo que una fenomenología trascendental no podría superar.

Ya en 1943, el propio Sartre presenta precisamente un supuesto "escollo del solipsismo" como una de las etapas de su tratamiento de la intersubjetividad en El ser y la nada, dando, así, continuidad a una preocupación que surgirá por vez primera en La trascendencia del ego, de 1936. En la formulación de El ser y la nada, el carácter profundamente paradójico de la experiencia del otro está contenido en la propia idea de una experiencia por la cual se da algo que debe comparecer delante de mí como un objeto de experiencia que anula mi propia experiencia, en cuanto su presencia para mí significa la emergencia de un sujeto que apunta a mi propia constitución como objeto. El surgir de otro sujeto en mi experiencia es mi devenir objeto para el otro y tener conciencia de mí mismo como objeto por la mediación de otra conciencia. En las propias palabras de Sartre,

El otro se presenta [...], en un cierto sentido, como la negación radical de mi propia experiencia, pues él es aquel para quien yo no soy sujeto, sino objeto. Me esfuerzo,

\footnotetext{
1 "Wenn Ich, das meditierende Ich, mich durch die phänomenologische epoche auf mein absolutes transzendentales Ego reduziere, bin ich dann nicht zum solus ipse geworden, und bleibe ich es nicht, solange ich unter dem Titel Phänomenologie konsequente Selbstauslegung betreibe? Wäre also eine Phänomenologie, die Probleme objektiven Seins lösen und schon als Philosophie auftreten wollte, nicht als transzendentaler Solipsismus zu brandmarken?". Edmund Husserl, Gesammelte Werke-Husserliana ILX, Dordrecht, Springer (con anterioridad: Den Haag, Martinus Nijhoff; Dordrecht / Boston / London, Kluwer Academic Publishers), 1950-2009, Hua I, p. 91.
} 
por tanto, como sujeto del conocimiento, por determinar como objeto al sujeto que niega mi carácter de sujeto y que me determina él mismo como objeto. ${ }^{2}$

De dar crédito a estos dos autores, que son referencias mayores en la construcción de una teoría fenomenológica de la intersubjetividad, todo sucede como si cualquier teoría fenomenológica de la existencia del otro tuviese que presentarse como resolución de una pretendida crisis solipsista implícita a la propia fenomenología. No en tanto, viendo de cerca, juzgamos poder afirmar que ni el problema del solipsismo tiene igual naturaleza y función en los dos autores, ni las soluciones buscadas convergen a un mismo objetivo. Es justamente en ese contexto de una aproximación de los dos fenomenólogos -atenta, por lo tanto, a las diferencias y a los eventuales fracasos de una y otra comprensión del problema del solipsismo y del fundamento de la relación intersubjetiva- que, en lo que sigue, examinaremos la teoría sartreana del être-pour-autrui, del ser-paralos-otros, y la teoría husserliana de la Einfühlung, de la "intropatía" (o, para utilizar una expresión más corriente, de la empatía), en cuanto forma de fondo de la experiencia de otro sujeto.

\section{2. ¿HAY UN PROBLEMA FENOMENOLÓGICO DEL SOLIPSISMO?}

Antes, sin embargo, es necesario esclarecer en que sentido hay, para la fenomenología, algo así como un problema del solipsismo y, lo que es más importante, en que sentido el problema clásico del solipsismo no es un problema que la fenomenología deba tener ni siquiera en consideración.

En primer lugar, la fenomenología no es una inquisición de tipo argumentativo. Del mismo modo que ella no produce ninguna prueba de la existencia del mundo, a la manera cartesiana, tampoco se puede encontrar en ella un argumento cualquiera que apuntase a probar la existencia de otro sujeto. La existencia, en general, no se demuestra -se verifica. Las lecciones humeana y kantiana siguen siendo enteramente actuales. Y la Fenomenología, en particular, debido a su orientación descriptiva y a su concentración temática en la estruc-

2 "Autrui [...] se présente, en un certain sens, comme la négation radicale de mon expérience, puisqu'il est celui pour qui je suis non sujet mais objet. Je m'efforce donc, comme sujet de connaissance, de déterminer comme objet le sujet qui nie mon caractère de sujet et me détermine lui-même comme objet". L'être et le néant, Paris, Gallimard, 1943, p. 273. 
tura de sentido de los actos intencionales, no pretende producir ninguna prueba que nos asegure para siempre la existencia del mundo y de otros sujetos en el mundo, sino explicitar el sentido de los actos intencionales en que otro sujeto es mentado y puesto como existente. El otro no es, para la fenomenología, una tesis a demostrar, como lo había sido para Descartes -es un sentido a describir. Y un sentido de una experiencia que es siempre "mía", sea quien sea el que, ahí, se diga "yo" a sí mismo.

Las cuestiones típicas de la fenomenología no son, pues, cuestiones sobre la existencia o no existencia de los objetos de los que podemos tener experiencia, sino sobre el sentido de la experiencia de esos objetos. Esta conversión temática es el propio ejercicio de la reducción fenomenológica. Sea lo que sea el mundo en sí mismo -y esa determinación de la estructura de la realidad es el campo, que la fenomenología no disputa, de las ciencias empíricas de la Naturaleza-, sea lo que sea el mundo objetivamente considerado, la cuestión fenomenológica no versa sobre el mundo que actualmente es, el cual es puesto "entre paréntesis" y entendido como un caso de una variación infinita de mundos posibles, sino antes sobre la experiencia por la cual hay conciencia de mundo. Así, la fenomenología, en cuanto analítica de la intencionalidad de la conciencia, no está, desde el principio, comprometida con ningún cuestionamiento ontológico ni con ninguna afirmación de existencia. En particular, ella no es el lugar donde la creencia natural en la existencia de otros sujetos se puede trasformar en una definitiva certeza. Para la fenomenología, la cuestión, la única cuestión que ella puede formular temáticamente, es indagar al respecto de los actos intencionales cuyo sentido es "experiencia de otro sujeto", intentar dilucidar su estructura interna y, eventualmente, las formaciones de sentido que los fundan y que funcionan, relativamente a ellos, como un presupuesto según el régimen de la "pre-donación" (Vorgegebenheit).

Se percibe así en que sentido Husserl puede afirmar, al inicio de su quinta meditación, que la fenomenología trascendental hasta ahí desenvuelta daba la espalda a la "objeción del solipsismo". No se trataba de que la Fenomenología trascendental volviese incierto nuestro saber acerca de la existencia de otros sujetos. Tal y como la fenomenología no subvierte la certeza del mundo, sino que la pone entre paréntesis para concentrarse no en el mundo que actualmente es, sino en el fenómeno-mundo, en cuanto estructura a priori que hace posible la experiencia y el conocimiento de cualquier mundo, actual o posible, tam- 
poco la fenomenología introduce ningún tipo de incerteza sobre nuestra convicción natural acerca de la existencia actual de otros sujetos (poner entre paréntesis no es poner en duda). Se trataba antes, para Husserl, con la invocación de la "objeción del solipsismo", de determinar si la fenomenología, tal y como había sido desenvuelta hasta el final de su cuarta meditación, hacía ya visible el sentido de la experiencia "otro sujeto", tanto en su génesis como en su específica configuración de sentido. Bien entendido, la respuesta sólo podía ser negativa: la teoría de la constitución de objetos de todo tipo, que había sido hasta ahí desenvuelta, tenía como límite el caso en que el objeto constituido era otro sujeto, o sea, no un puro polo sintético de unidad de la multiplicidad de mis experiencias, sino un centro autónomo de organización de otra vida de conciencia. En una palabra, el punto paradójico era, aquí, que el objeto constituido era, él mismo, un sujeto, para el cual el sujeto constituyente se convertía en un objeto en el mismo movimiento que lo constituía. Para esta reversibilidad, la fenomenología de la constitución objetual desenvuelta hasta la cuarta meditación no tenía aún instrumentos analíticos a su disposición. Sino que, más importante todavía, esta capa superior de la constitución alteraba en retrospectiva los propios resultados antes alcanzados, pues ella debería mostrar como la constitución de la objetividad en general era ya una realización no de un ego solitario (el único, en el estadio de desenvolvimiento de la fenomenología trascendental hasta entonces alcanzado), sino de una comunidad intersubjetiva, para la cual la capa del ser-objetivo se constituía sobre la forma de un mundo común (Gemeinwelt) accesible para cualquiera (für jedermann zugänglich). He ahí la razón por la que la fenomenología desenvuelta hasta la cuarta meditación daba la espalda a la objeción solipsista: no sólo el modelo de la constitución objectual (polo sintético de una multiplicidad de actos intencionales) era insuficiente para la teoría de la intersubjetividad, como la propia constitución de objetos en ese primer sentido presuponía la constitución intersubjetiva, en la medida en que "objeto", según su sentido auténtico, significaba un polo de validaciones intersubjectivas. En su forma sistemática, las cuestiones de la intersubjetividad y de la comunidad intersubjetiva venían, pues, antes que las cuestiones relativas a la constitución objectual en general. He ahí la razón de la situación incómoda en que la fenomenología estaba colocada en la cuarta meditación.

La crisis solipsista de la fenomenología de Husserl está, por lo tanto, en un lugar diferente de aquel en que sus críticos la creen encontrar. Se dice común- 
mente que, para la fenomenología trascendental de Husserl, sólo la certeza del ego es apodíctica, en cuanto que la conciencia de un mundo y la de otros sujetos en el mundo es, y para siempre será, una simple verosimilitud. Pero esta forma de argumentar es una burda mistificación. Ciertamente que la descripción de la experiencia de varias categorías de objetos, realizada por la fenomenología, cualifica también el valor epistémico de esa experiencia. Así, la autocerteza de un ego es apodíctica (aunque no adecuada) porque no es posible, por razones de esencia, que alguna vez se pueda verificar una discordancia entre el acto por el cual un ego se dirige a sí mismo y la intuición de sí mismo en cuanto existente. La conciencia de la existencia propia no está abierta a la Täuschung, o sea, al error o a la decepción. Ya la conciencia de los objetos en el mundo y de otros sujetos en el mundo está constantemente expuesta a la posibilidad de que la intención que los menta no sea confirmada, sino negada por el plexo de intuiciones que vienen a llenar esa intención. En una palabra, la experiencia de otro sujeto está, por esencia, siempre abierta al error y a la rectificación. La diferencia que separa una y otra es la diferencia entre los tipos de intuición. Es en ese sentido que la autocerteza del ego es apodíctica y que la conciencia de cualquier objeto del mundo es siempre conciencia de una existencia para siempre probable, que las percepciones siguientes pueden sucesivamente reforzar, sin elevarla jamás, todavía, al plano de la intuición adecuada y de la evidencia apodíctica. Pero que los objetos de una experiencia sean dados como contingentes no significa que la experiencia de esos objetos sea, ella misma, un hecho contingente. Al contrario, la experiencia de un mundo y de otros sujetos en y para el mundo es un ingrediente de la experiencia en general y no tiene ningún sentido hablar de un ego destituido de la experiencia de un alter-ego y de un mundo natural como estrato subyacente. Es este error categorial el que tiene que ser extirpado.

\section{TRES CUESTIONES SOBRE LA CONCIENCIA INTERSUBJETIVA}

"Otro sujeto" es, pues, fenomenológicamente, un sentido a describir, no una existencia a demostrar. Es un objeto reducido, entre aspas, y no una objetividad trascendente, dada en actitud natural, por mucho que incumba también a la descripción fenomenológica la explicitación del modo como la existencia de 
otro sujeto puede ser dada y comprobada. Se trata, sin embargo, de un problema eidético, acerca del modo en que la intención significativa obtiene su cumplimiento en una intuición, y de qué tipo es esa intuición que permite, por esencia, cualquier posición racional posible de la existencia de un alter-ego. En la fenomenología, no podemos encontrar, entonces, respuestas a cuestiones sobre la existencia actual de otros sujetos en el mundo (si bien encontramos cuestiones acerca de la posibilidad de la posición racional de la existencia de otro-yo, en cuanto legalidad eidética) o de comunidades de vida cultural y histórica, sino respuestas a cuestiones que tienen que ver con el significado y la génesis del sentido alter-ego en cuanto ingrediente de la experiencia de un mundo. Para la fenomenología trascendental, la cuestión no versa sobre una multiplicidad en-sí de subjetividades, y sobre las leyes históricas, sociales, biológicas, que en-sí regulan esa multiplicidad, sino antes bien sobre el sentido de la experiencia de la intersubjetividad y sobre el sentido de la experiencia de una comunidad de sujetos, entendidas eidéticamente, o sea, como legalidades de esencia a establecer independientemente de cualquier cuestión fáctica que sea determinada por la posición previa de una existencia actual. Toda y cualquier existencia actual es, al contrario, metódicamente puesta entre paréntesis por la reducción trascendental fenomenológica.

En este contexto, las cuestiones fenomenológicas más básicas (olvidando las importantes cuestiones al respecto de la forma racional de una vida y de una comunidad "auténticas") son del siguiente tipo:

1. ¿Cuál es el contenido de sentido de la aprehensión de un alter-ego, en su estructura noético-noemática? O sea: ¿qué significa aprehender algo en cuanto otro sujeto?

2. ¿Qué motivación conduce a un ego a la experiencia de un alter-ego? 0 sea, ¿cuál es el tipo de "impulso" (¿habrá ahí una Triebintentionalität?) que conduce a un ego a la posición y la experiencia de otro ego?

3. ¿Cuál es la génesis del sentido alter, o, más específicamente, del sentido "otro" bajo la forma "otro-yo"? ¿Es el otro yo la simple multiplicación proyectiva de mi propio ser, ya pre-dado de antemano e inalterado en la aprehensión subsiguiente del otro, o un ingrediente constitutivo de la propia experiencia de mí mismo?

Son estas cuestiones más básicas sobre el significado de la experiencia de un alter-ego y sobre la propia génesis del sentido alter que nos sirvieron de 
hilo-conductor en el análisis de los conceptos de ser-para-los-otros y de empatía, de Sartre y de Husserl. Un ego es un ser en auto-aparición, o sea, un ser cuyo aparecer es aprehendido como suyo. ¿De qué modo el aparecer del otro, o sea, el aparecer aprehendido bajo el modo otro-yo, se viene a conectar con la aparición bajo el modo auto? Incluso más, de qué modo un ser en autoaparición se modifica en un ser que aparece a otro, o sea, como una autoaparición se puede modificar en hetero-aparición, y de que modo esa heteroaparición actúa en contrapartida sobre el propio sentido de la auto-aparición -tales son las cuestiones.

\section{LA ESTRUCTURA FUNDAMENTAL DE LA CONCIENCIA DEL OTRO}

La idea fundamental de Sartre es la de que (a) conciencia de otro sujeto y (b) conciencia de sí como objeto son equivalentes. O sea, tener conciencia del Otro y tener conciencia de sí mismo como objeto (para Otro) son estructuras superponibles. Conciencia de Otro es, pues, el proceso por el cual devengo un ser (para-Otro), un être-pour-autrui, de tal modo que la llave de la descripción fenomenológica del sentido "otro-sujeto" es dada por la descripción del modo como se produce una modificación en la autoconciencia que va del para-sí (pour-soi) a la autoconstitución como un "ser" (en-soi), captable en una conciencia (auto) objetivante, cuya hipóstasis es, precisamente, mi propia contraposición a una alteridad para la cual soy ese objeto. Así, captar al otro es captarme en el modo como soy objeto para su conciencia. Pero, ¿cómo captamos precisamente esa otra conciencia? Nos encontramos a la puerta de una paradoja: para captar el modo como somos objeto para otra conciencia es necesario captar esa conciencia para quien somos objeto, pero captarla es, a su vez, captar el modo como somos objeto para ella, y así sucesivamente, en una circularidad sin salida o en un perpetuo juego de espejos en que tenemos, infinitamente, las múltiplas reverberaciones de la conciencia de nosotros mismos. En un cierto sentido esencial, el problema de la alteridad es formulado y tratado en el horizonte del problema de la autoconciencia: hay un cierto régimen de la autoconciencia que realiza la posición de una alteridad, y esa posición de la alteridad como momento de esa autoconciencia es la descripción del sentido alter- 
ego. He ahí la intuición fundamental de Sartre. Son muchos los lugares en los que esta dirección se anuncia. Por ejemplo,

No puedo ser objeto para mí mismo pues soy lo que soy; abandonado a mis recursos propios, el esfuerzo reflexivo para el desdoblamiento conduce al fracaso, soy siempre retomado por mí mismo. Y cuando afirmo ingenuamente que es posible que yo sea, sin darme cuenta, un ser objetivo, supongo implícitamente, con eso, la existencia de Otro, pues, ¿cómo sería objeto, a no ser para un sujeto? Así, el Otro es primero, para mí, el ser para el cual soy objeto, o sea, el ser por el cual gano mi objetualidad. ${ }^{3}$

Esta idea central de Sartre es, además, presentada de un modo tan sutil, que muchas veces ella es casi ocultada en las largas explicaciones de El ser y la nada. De hecho, es preciso navegar verdaderamente entre Cila y Caríbdis (como el texto arriba lo hace) cuando, por un lado, se parte de la tesis según la cual (a) la conciencia de otro sujeto es dada por la (auto)conciencia de mi propia "objetualidad" (o de mí mismo como un en-soi), mas, por otro lado, para evitar la consecuencia de hacer del sentido "otro sujeto" un momento necesario, apriorístico, de la autoconciencia y, así, dar una solución puramente verbal (de hecho, una pseudo-solución) al problema del solipsismo, se pretende conjugar esa tesis con la afirmación de que (b) la existencia de El otro es un hecho contingente, de tal modo que el ser-para-el otro no surja como un elemento estructural del para-sí. O sea, si la autoconciencia como objeto es equivalente a la conciencia de otro sujeto, el otro no es, sin embargo, un puro mediador entre mí y mí (o el sentido hipostasiado de esa mediación), porque sólo delante de la presencia fáctica del otro será posible por sí mismo ese modo de autoconciencia. Sólo la contingencia del otro puede, en verdad, asegurar que el ser-parael-otro sea un modo de existencia que el para-sí sólo asume delante del encuentro concreto, puramente contingente, con otra conciencia.

Así, la verdadera tesis de Sartre es que (c) la autoconciencia objetivante (o la conciencia de mi propia "objetualidad") es un modo de conciencia de mí mismo que sólo me es accesible delante de la presencia fáctica de otro sujeto (modo que no equivale, por tanto, a la intensificación de la simple escisiparidad reflexiva):

\footnotetext{
3 "Je ne puis être objet pour moi-même car je suis ce que je suis; livré à ses seules ressources, l'effort réflexif vers le dédoublement aboutit à l'échec, je suis toujours ressaisi par moi. Et lorsque je pose naïvement qu'il est possible que je sois, sans m'en rendre compte, un être objectif, je suppose implicitement par là même l'existence d'autrui, car comment serais-je objet si ce n'est pour un sujet ? Ainsi autrui est d'abord pour moi l'être pour qui je suis objet, c'est-à-dire l'être par qui je gagne mon objectité". Jean-Paul Sartre, op. cit., pp. 316s.
} 
[...] el ser-para-el otro no es una estructura ontológica del para-sí. [...] No sería, tal vez, imposible concebir un para-sí totalmente libre de todo y cualquier para-el otro y que existiese incluso sin sospechar acerca de la posibilidad de ser objeto. ${ }^{4}$

La existencia de los otros no es, en efecto, una consecuencia que pueda deducirse de la estructura ontológica del para-sí. Es un acontecimiento primero, ciertamente, mas de orden metafísico, o sea, que habla al respecto de la contingencia del ser. ${ }^{5}$

No en tanto, como el encuentro fáctico con otro sujeto es siempre descrito como ese devenir consciente de sí como objeto, como lo muestran los penetrantes análisis del fenómeno de la vergüenza (honte) como estructura fundante de la conciencia de la alteridad, la paradoja de que hablamos antes se mantiene intacta: la presencia del otro significa hacer-me autoconsciente de mi ser objectual, y ser consciente de mi ser-objeto es la aparición o la presencia de otro sujeto, "es en la y por la revelación de mi ser-objeto para el otro que debo poder captar la presencia de su ser-sujeto" ${ }^{\prime 6}$.

[...] El otro es el mediador indispensable entre mí y mí mismo: tengo vergüenza de mí tal como aparezco a el otro. Y, por la propia aparición del otro, permanezco en condiciones de emitir un juicio sobre mí mismo como un objeto, pues es como objeto que aparezco al otro. ${ }^{7}$

Las figuras del otro en su presencia, en sus motivaciones, en sus intenciones, son recubiertas por las múltiples formas de la conciencia de sí mismo delante de él. Si el otro está ahí mismo presente $-\mathrm{y}$ Sartre, atento al supuesto "obstáculo solipsista", siempre hará de la conciencia de otro sujeto la posición de una alteridad irreducible-, los análisis de Sartre siguen, todavía, en la dirección de mostrar que su presencia es el lugar para un replanteamiento temático de mí mismo, en que el se vuelve el mediador para una nueva forma de mi propia autoconciencia, precisamente la que me revela (o sea, me muestra por primera vez) mi propio ser como una "naturaleza". La motivación que me conduce al otro es, aún y siempre, el cuidado de mí mismo. Tal es la consecuencia.

4 "[...] L'être-pour-autrui n'est pas une structure ontologique du pour-soi. [...] Il ne serait peut-être pas impossible de concevoir un pour-soi totalement libre de tout pour-autrui et qui existerait sans même soupçonner la possibilité d'être un objet". Ibidem, p. 329.

5 "L'existence des autres n'est pas, en effet, une conséquence qui puisse découler de la structure ontologique du pour-soi. C'est un événement premier, certes, mais d'ordre métaphysique, c'est-à-dire qui ressortit à la contingence de l'être". Ibidem, p. 344.

6 "C'est dans et par la révélation de mon être-objet pour autrui que je dois pouvoir saisir la présence de son être-sujet". Ibidem, p. 302 (subrayados nuestros).

7 "[...] Autrui est le médiateur entre moi et moi-même: j'ai honte de moi tel que j'apparais à autrui. Et, par l'apparition même d'autrui, je suis mis en mesure de porter un jugement sur moi-même comme sur un objet, car c'est comme objet que I'apparais à autrui". Ibidem, p. 266. 
En el límite, el comportamiento humano y toda la tela compleja de las relaciones intersubjectivas no será comportamiento motivado por el otro, con y para el otro, sino comportamiento motivado por el modo como nos captamos como objeto para el otro. Este resultado, implícito en la teoría sartreana de la conciencia de otro sujeto, amenaza con hacer del ego, más que un yo solipsista, un yo que hace caer las relaciones intersubjectivas en un sombrío cuidado obsesivo consigo mismo, que vuelve difícil concebir la propia posibilidad de lazos de socialización construyéndose bajo la forma superior de sentido del "nosotros". Alias, consecuente con su crítica del Mitsein heideggeriano ${ }^{8}$, Sartre dirá que el "nosotros" o es un nosotros-objeto, por tanto, una formación sólo visible desde el exterior, o, cuando se trata del nosotros-sujeto, será un fenómeno puramente psicológico sin consistencia ontológica9. Al conducir el análisis de la relación intersubjetiva según el patrón del ser-para, o sea, de una cierta forma de la relación cara-a-cara que se determina como confrontación (o afrontamiento) de dos libertades, recíprocamente sujeto y objeto una para la otra, la construcción de un mundo común y de una comunidad constituyéndose en procesos implicando personalidades de "orden superior", en la forma fundada del "nosotros", acaba por perderse completamente del horizonte. Dicho con otras palabras: el círculo en que las libertades son recíprocamente sujeto y objeto una para la otra ( $y$, por tanto, se destituyen una a la otra como libertades) no es jamás roto en dirección a un punto en que ambas serían sujetos para un mismo mundo común. Mas éste es un resultado que anticipa conclusiones que sólo más adelante se volvieron enteramente visibles.

\section{INTERSUBJETIVIDAD Y REFLEXIÓN}

El punto esencial es, sin embargo, el significado ontológico del ser-para-elotro y el modo como se entrelaza con las formas de cuasi auto-objetivación del para-sí, sobre todo la forma reflexiva. Según los penetrantes análisis de Sartre, este devenir objeto para el otro produce una dramática alteración del modo de ser del existente humano: en la medida en que se objetiva para el otro (o tiene

\footnotetext{
${ }^{8}$ Ver ibidem, pp. 290ss.

${ }^{9}$ Ver ibidem, pp. 464ss.
} 
conciencia de sí mismo por medio de la conciencia de su devenir-objeto para el otro), adquiere la consistencia de un ser, su libertad es trascendida en la trascendencia de otro sujeto y él mismo se revela según el modo de ser del en-soi. Sartre lo dice impresionantemente en El ser y la nada:

\begin{abstract}
Mas este ser [en cuanto visto por el otro], la vergüenza me revela que yo lo soy. No bajo el modo del haber-sido o del "tener que ser", mas en-sí. [...] Una vez más, el ensí se forma de nuevo sobre el para-sí. Mas, una vez más, esta metamorfosis se opera a distancia: para el otro, yo estoy sentado como este tintero está sobre la mesa: para el otro, estoy inclinado sobre el agujero de la cerradura tal como este árbol está inclinado al viento. Así, yo me despojé para el otro de mi trascendencia, [...] yo tengo un exterior, una naturaleza, mi caída original es la existencia del otro. ${ }^{10}$
\end{abstract}

Las adjetivaciones sartreanas de esta alteración profunda de la estructura ontológica de la realidad humana hablan por sí: "El otro me alienó de mí mundo", "ser visto implica la alienación del mundo que yo organizo", "el otro es la muerte escondida de mis posibilidades en cuanto yo mismo vivo esa muerte", "mi ser para-el otro es una caída, [...] y esta caída es alienación"11. De ahí que, para Sartre, asistamos, a propósito de la intersubjetividad, a una dialéctica irresoluble entre ser-objeto para el otro y constitución del otro como objeto. Es el afrontamiento, directo, de dos libertades y, más allá de eso, la tentativa, fracasada, de cada una de ellas de absorber a la otra. El sentido fundamental de esta dialéctica es el conflicto: "el conflicto es el sentido originario del ser-parael-otro". ${ }^{12}$

Para comprender bien lo que aquí está en juego, retrocedamos un poco. En La trascendencia del ego, Sartre niega la tesis de que el yo esté formal o materialmente en la conciencia. La conciencia pre-reflexiva no dice "yo". El yo está antes en el mundo, es un ser mundano tanto como el yo de los otros. Ciertamente que el yo se da a la conciencia trascendental como íntimo. Pero eso no significa que sea el sujeto de la conciencia -el yo no es de la conciencia; hay, antes, un yo para la conciencia. Según Sartre, el campo trascendental del que habla la fenomenología de Husserl debe ser caracterizado como impersonal, o mejor, como pre-personal, o sea, sin yo. El yo es, como las otras cosas del

\footnotetext{
10 "Mais cet être, la honte me révèle que je le suis. Non pas sur le mode de l'étais ou du 'avoir à être', mais en-soi. [...] Mais, une fois de plus, cette métamorphose s'opère à distance: pour l'autre, je suis assis comme cet encrier est sur la table; pour l'autre, je suis penché sur le trou de la serrure, comme cet arbre est incliné sur le vent. Ainsi ai-je dépouillé, pour l'autre, ma transcendance. [...] J'ai un dehors, j'ai une nature; ma chute originelle c'est l'existence de l'autre". Ibidem, pp. 308s.

${ }^{11}$ Ibidem, pp. 307, 309, 311, 321, respectivamente.

12 "Le conflit est le sens originel de l'être-pour-autrui". Ibidem, p. 413.
} 
mundo, un objeto para la conciencia. Es una unidad noemática, no una unidad noética. La conciencia es un absoluto, en la medida en que es conciencia de sí en cuanto conciencia de un objeto. Mas este "sí" de la conciencia se refiere a una distancia pre-reflexiva que aún no es la diferencia entre un yo y sus actos. Se trata de una distancia entre, por ejemplo, creencia y conciencia de creencia, una distancia que es constitutiva de la propia conciencia irreflexiva, en cuanto ella es conciencia de ser conciencia de un objeto cualquiera. Como Sartre dirá más tarde, la conciencia está a distancia de sí misma por una diferencia que es un néant. Así, toda conciencia es conciencia de su objeto y de sí misma en cuanto conciencia de ese objeto. Tal es el plano pre-reflexivo. La reflexión que Sartre designa como "impura" es el lugar de constitución del ego. Es el acto reflexivo el que pone por vez primera un yo "por detrás" del acto reflexivo a título de su sujeto. El ego es, así, constituido, en la reflexión impura, como sujeto de los estados y cualidades (se trata del Moi) y de las acciones (se trata del Je). Esta constitución del yo como punto virtual de unidad del psiquismo es -así reza la interpretación "existencial" de Sartre acerca de este movimiento "auto-ilusorio" por el cual la conciencia se identifica con el ego que ella constituye- el momento en que la conciencia niega su propia libertad y se comprende como un ser, que tiene sus propiedades fijas, sus cualidades, sus estados, sus acciones, en fin, una naturaleza. La constitución del yo es, por eso, según las consideraciones finales de La trascendencia del ego, una estrategia de huída a la angustia de una libertad "monstruosa", "insoportable", que caracterizaría a la conciencia en cuanto existente absoluto, sin porque y sin razón:

todo sucede como si la conciencia constituyese al ego como una falsa representación de sí misma [...] La conciencia, advirtiendo de lo que se podría llamar la fatalidad de su espontaneidad, se angustia en el mismo momento [...] Si el Yo del Yo pienso es la estructura primera de la conciencia, esta angustia es imposible. ${ }^{13}$

Según Sartre, esta teoría resolvería definitivamente el problema del solipsismo con el que la fenomenología egológica de Husserl se habría confrontado. Pues no se trataría ya de saber como es que un yo conoce a otro yo, sino apenas de mostrar que el yo propio y el yo ajeno están ambos en el mundo como objetos de la conciencia absoluta y que son tan ciertos el uno como el otro. Mas

13 "Tout se passe donc comme si la conscience constituait l'Ego comme une fausse représentation d'elle-même [...] Alors la conscience, s'apercevant de ce qu'on pourrait appeler la fatalité de sa spontanéité, s'angoisse tout à coup [...]. Si le Je du Je pense est la structure première de la conscience, cette angoisse est impossible". La transcendance de l'ego, Paris, Vrin, 1981, pp. 82s. 
esto no resuelve el problema, como Sartre bien advirtió en El ser y la nada. Por el contrario, lo profundiza y lo hace intratable. Pues ahora es el propio campo trascendental pre-personal de cada conciencia el que es absolutamente solitario y sin mediación posible con otro campo trascendental. Un yo puede bien aparecer y ser aprehendido por el otro. Pero un campo trascendental puro es una interioridad que, por definición, no tiene exterior.

Contra esta solución demasiado optimista de La trascendencia del ego, Sartre ve en los análisis del ser-para-el-otro de El ser y la nada una respuesta finalmente satisfactoria al problema. No en tanto, vista de cerca, esta nueva posición de Sartre es estructuralmente idéntica a la que había sido desenvuelta en La trascendencia del ego, pues se basa todavía en la idea de una autoconstitución de la conciencia como un ser o un en-soi. La diferencia notable es que este en-soi no es simplemente el resultado de la reflexión impura, sino de una, por así decir, hetero-reflexión, en que la conciencia del otro, en su irreductible alteridad, garante la constitución de un en-soi que ya no puede ser retomado y absorbido por el para-sí. Todo sucede como si esa autoconstitución como un ser, para mantenerse, careciese, más que de la distancia entre reflexionado y reflexión, de la distancia entre conciencia propia y conciencia ajena. Lo que la reflexión (impura) no puede realizar completamente, lo realiza esa reflexión "a la segunda potencia" que es la conciencia del otro y mi reflexión especular en ella. Sartre, a pesar de esta alteración, continua viendo la génesis del sentido "otro sujeto" como algo que surge en la prolongación de la reflexión. Así, el sentido "otro" es todavía y siempre reconducido a la conciencia de sí.

Ahora bien, esto es una base incorrecta para una teoría de la intersubjetividad, pues la conciencia de otro sujeto es algo que no depende de la reflexión y que incluso le es anterior. El propio Sartre admite que el ser-para-el otro es comprendido, en El ser y la nada, en la extensión de la reflexión y como una especie de reflexión a la segunda potencia: "la ec-stasis reflexiva se encuentra a camino de una ec-stasis más radical: el ser-para-otro. [...], [...] en esta tercera ec-stasis, asistimos a algo como una escisiparidad reflexiva más desenvuelta, [...] el ser para el otro parece ser la prolongación de la pura escisiparidad reflexiva". ${ }^{14}$ De este modo, el proceso de auto-aprehensión como un

\footnotetext{
14 "Ainsi l'ek-stase réflexive se trouve sur le chemin d'une ek-stase plus radicale: l'être-pour-autrui. [...] Dans le cas de la troisième ek-stase, nous assistons comme à une scissiparité réflexive plus pous-
} 
ser y de denegación de la libertad no es apenas característico de la reflexión impura. El conoce en la posición del otro su momento conclusivo. No es apenas para mí que me constituyo como un ser -es en la conciencia del otro para mí (dada en el fenómeno del regard) que ese proceso de cosificación alcanza su acabamiento final. Así, la conciencia del otro es pura potenciación de la reflexión, ella intensifica la dualidad reflexiva y le añade una segunda negación interna, que la vuelve irresoluble, pues, ahora, resolverla sería suprimir la alteridad de otra conciencia.

Es precisamente por eso por lo que, como vimos, Sartre puede, sin la sombra de una duda, tratar la conciencia del otro como si ella fuese equivalente a la conciencia de mí mismo para una otra conciencia, o sea, como si el otro fuese apenas una ocasión y un suporte para la conciencia de mí mismo. En verdad, lo que vemos no es al otro, sino nuestra reflexión especular en él. Tener conciencia de otro sujeto es, sartreanamente, vernos por la hipóstasis de otra conciencia. Que la relación intersubjetiva sea un perpetuo juego de espejos, que, en la captación de otro sujeto, apenas nos sea devuelta una vez más apenas nuestra propia imagen en una última degradación, o sea, como objeto, he ahí el resultado poco satisfactorio, mas, con todo, inevitable de la comprensión sartreana de la dinámica de la intersubjetividad. El sentido "otro" es apenas el punto virtual que sustenta ese último ver sobre mí mismo. Es una ocasión, y la conciencia es la soledad del soi-même y de las múltiples reverberaciones de ese su ser - primero, en la escisiparidad reflexiva, después, en la escisiparidad intersubjetiva. Y porque la escisiparidad intersubjetiva no podría ser reunificada sin una reabsorción de la libertad del otro que lo destruiría en su alteridad, cosa que es en sí misma contradictoria, he ahí el conflicto que emerge, como vimos, como fondo permanente de todas las relaciones humanas:

mi proyecto de recuperación de mí es, fundamentalmente, proyecto de reabsorción del otro. [...] Sería, por tanto, necesario [...] actuar sobre la negación interna por la cual el otro trasciende mi trascendencia y me hace existir para el otro, o sea, actuar sobre la libertad del otro" -"la unidad con el otro es, por tanto, irrealizable. [...], y este proyecto de unificación es fuente de conflicto. ${ }^{15}$

sée. [...] L'être pour autrui paraît être le prolongement de la pure scissiparité réflexive". L'être et le néant, pp. 346s.

15 "Ainsi, mon projet de récupération de moi est fondamentalement projet de résorption de l'autre [...] Il serait donc nécessaire [...] d'agir sur la négation interne par quoi autrui transcende ma transcendance et me fait exister pour l'autre, c'est-à-dire d'agir sur la liberté d'autrui". "L'unité avec autrui est donc, en fait, irréalisable. [...] Ce projet d'unification est source de conflit". Ibidem, pp. 414s. 
Al conducir la cuestión la intersubjetividad al horizonte de la reflexividad (o sea, de la conciencia tética de sí) y como escisiparidad hetero-reflexiva (petrificada por la mediación de otra conciencia), la conciencia de sí aparece como el círculo en que fatídicamente siempre nos encerramos, incluso cuando pretendemos asistir a la génesis del sentido "alter-ego".

Hay dos cuestiones de naturaleza diversa de las que una teoría de la intersubjetividad se debe ocupar. Una es: ¿cómo "veo" a otro sujeto y tengo conciencia de su existencia? Otra es: ¿cómo "me veo" en la conciencia que tengo de otro sujeto? Sartre respondió a la segunda creyendo que respondía a la primera. De ahí todas las ambigüedades e insuficiencias de su posición.

\section{LA APREHENSIÓN DEL OTRO COMO PROCESO PASIVO -CUERPO, SOMA Y PSIQUE}

Si queremos obtener una clarificación de la primera cuestión a que aludimos, tendremos que desplazarnos a la teoría husserliana de la Einfühlung, algo que no significa decir que esta teoría sea completa en sí misma y que represente la última palabra en lo que al tratamiento de la cuestión se refiere. Al contrario de la intuición fundamental de Sartre, la tesis de Husserl es que la conciencia de otro sujeto no es un acto de auto-aprehensión por la mediación de otra conciencia, sino una operación directa de transposición por analogía. O sea, la conciencia del otro no es aprehensión de mi devenir objeto para otro, no es recuperación de esa última extensión de mí mismo, sino recorte, sobre los objetos del mundo circundante, de un conjunto de señales que se organizan bajo la forma de un comportamiento y que, por eso, son interpretados por analogía con mi propio ser según un proceso pasivo que es, por tanto, anterior a cualquier conciencia temática de mí mismo. Cuando esto se verifica, algo aparece para mí en el mundo con el sentido "otro yo".

Para comprender bien esta génesis del sentido alter-ego, es necesario recordar el gran descubrimiento que alimenta las reflexiones de Husserl -la conciencia en que el ego trascendental hace experiencia del mundo es la conciencia por la cual el ego se pone a sí mismo en el mundo y se aparece a sí mismo como una unidad no sólo psíquica (seelisch), sino también somática (leiblich). En reflexiones retomadas siempre de nuevo y jamás llevadas a un punto conclusivo, Husserl intenta circunscribir este proceso por el cual la conciencia trascen- 
dental, en su devenir realidad humana en el mundo, se aparece a sí misma como unidad de un cuerpo y de una psique.

El primer obstáculo a evitar es la comprensión dualista, supuestamente cartesiana, de esta duplicidad, como si se tratase de la simple yuxtaposición de un corpus y de una mens. Es precisamente aquí que interviene la distinción husserliana crucial entre el simple Körper y el Leib. Un cuerpo en el mundo circundante se constituye en el fluir concordante de las percepciones parciales -es un proceso de confirmación progresiva de la creencia y de variación constante en el flujo de la intuición. Aparece en referencia a un aquí y un ahora. Pero este aparecer de la corporalidad física en el decurso de la percepción no podría verificarse sin la constitución concomitante de mi propio cuerpo como lugar de la sensibilidad y como realidad automotora en la experiencia cinestésica correspondiente, o sea, como un cuerpo animado, en que el dualismo de la res extensa y de la res cogitans fue desde el inicio superado. Este cuerpo que se constituye como mío, en el cual yo domino (el verbo usado siempre por Husserl es walten), con el cual me muevo en la exploración del mundo circundante, este cuerpo dado en una percepción interna que jamás se puede transformar en un completo objetivarse y en un verse "por fuera", este cuerpo que no es, en el fondo, cuerpo para la conciencia, sino conciencia de tener cuerpo, es justamente lo que Husserl denomina Leib. La conciencia que va hacia el mundo y se aparece como realidad humana en el mundo tiene en su base esta autoconstitución somática de la subjetividad.

Muchos estratos podrían ser discriminados en este proceso de somatización, o sea, en esta conciencia de tener cuerpo. Husserl limitó drásticamente su programa de descripción de esta autoconstitución corporal del sujeto al concentrarse en el fenómeno de la senciencia externa (sobre todo, de la visión y del tacto) y en las cinestesias. Pese a todo, esa limitación no quita agudeza a sus análisis, sino apenas completud. La descripción husserliana de la experiencia de otro sujeto es, desde el inicio, polarizada por este tema de la autoconstitución somática del ego en cuanto sujeto humano. Solamente en la medida en que aparezco corporalmente en el mundo puede otro cuerpo de este mundo emerger también para mí como cuerpo de otro sujeto, y yo mismo para él en cuanto sujeto corporal en una reciprocidad cerrada. El concepto mayor de esta intencionalidad que abre el espacio de la intersubjetividad es, para Husserl, el de Paarung, emparejamiento. El emparejamiento es un proceso general de la 
génesis pasiva. Designa ese fenómeno por el cual un contenido reenvía a otro según una síntesis de analogía. Aplicado a la cuestión de la intersubjetividad, el emparejamiento circunscribe el fenómeno primitivo de la conciencia de otro sujeto. Cuando la percepción de simples cuerpos en el mundo circundante es el lugar de una transposición (Übertragung) de mi propia experiencia somática dada en la "introcepción", ahí se verifica, también, un fenómeno singular que es, digamos, la dimensión más primitiva (mas no la única) de la conciencia de otro sujeto -ese cuerpo delante de mí, dado en un flujo de simples percepciones, se vuelve lugar de apresentación (Appräsentation) de otra realidad psicosomática que es aprehendida por analogía con la experiencia originaria de mí mismo. En una palabra: en la percepción de ese cuerpo sobreviene la conciencia de otro sujeto y ese cuerpo, constantemente dado en el discurrir perceptivo, es, ahora, no simple cosa entre cosas, sino el lugar donde emerge para mí y se viene a expresar otra vida de conciencia, es un "cuerpo-somático" (Leibkörper), animado por una psique. En los movimientos de otro cuerpo, no se hacen ahora patentes sólo traslaciones físicas; esos movimientos son ahora las señales que expresan, por ejemplo, disposiciones de la voluntad, sentimientos, emociones, esto es, fenómenos de una vida psíquica. De tal modo que una expresión facial no es un simple fenómeno físico de contracción muscular o que un movimiento de un brazo no es un simple desplazamiento de un cuerpo en el espacio: son ahora señales, y señales que expresan que, en ese cuerpo, algo acontece que sólo puede ser interpretado a partir de la relación que yo establezco, en cuanto ego psíquico, con el cuerpo que originalmente surge como mío. Estos movimientos son ahora gestos expresivos, son comportamientos de una vida psíquica en su corporalización. La expresión facial y el movimiento largo del brazo no valen más por sí mismos, sino por lo que expresan: el dolor, la alegría, la impaciencia o la tristeza. La dimensión somática de la subjetividad es, así, la del cuerpo interpretado según el régimen de la expresividad. El cuerpo que se hace, así, cuerpo expresivo y que envía su corporalidad natural hacia una posición de simple fondo no-temático, sobre el cual se sobrepone la conciencia de una vida psíquica somatizada, es, por consiguiente, el modo originario de la irrupción de otro sujeto en mi experiencia del mundo. Éste despunta como otro centro de organización de un mundo circundante, a partir de su "aquí-ahora", mundo circundante que, en la conciencia de su sobreposición con el mío propio, hace emerger por vez primera el sentido "mundo común". 
En un manuscrito preparatorio de las Meditaciones cartesianas, Husserl muestra con claridad los niveles y el programa de la descripción del sentido alter-ego:

\begin{abstract}
Debe ser mostrado como la semejanza sensible del cuerpo "allí" con el cuerpo que, en cuanto cuerpo-somático, es portador del estrato de sentido de la animación, desde luego, de la somaticidad específica [...], y que es el objeto-cero del mundo que se aparece "orientado a su alrededor", permite hacer crecer, en la aprehensión de aquel cuerpo, el sentido "soma coexistente, en cuanto miembro-cero de un mundo propio primordialmente constituido, juntamente con el correspondiente ego y su vida de conciencia", todo esto, sin embargo, en cuanto co-existente "apresentado", en una presentificación vacía, que no puede asumir el carácter de la presentación efectiva, por consiguiente, de la originalidad de la donación efectivamente primordial. ${ }^{16}$
\end{abstract}

Esta conciencia de otro sujeto está, para Husserl, como es bien visible, fundada en el proceso originario por el cual constituyo somáticamente mi propia realidad mundana y sería imposible sin ésta. Es en esa medida que la percepción de otro sujeto es una transposición por analogía. No porque el otro sea la simple proyección especular de mí mismo, no porque vea al otro como una multiplicación de mí mismo, sino porque el otro, en su alteridad, sólo es visible por analogía con el modo en que yo mismo me sé como un sujeto psicosomático. A cada momento tenemos conciencia de esto: es casi imposible observar, por ejemplo, a alguien absorbido en un trabajo manual sin que interpretemos sus gestos como un comportamiento y sin que mentalmente casi reproduzcamos sus movimientos a partir de nuestra relación original con nuestro propio cuerpo y a partir del modo en que nuestras intenciones y vivencias dirigen las exteriorizaciones corporales a partir de una unidad no-somática (no-localizable somáticamente en la introcepción) que aprehendemos como "nuestro yo". En ciertos casos, como cuando observamos a alguien esforzarse en realizar determinada tarea (o sea, cuando aprehendemos a un sujeto psíquico suponiendo, en sus movimientos corporales, intenciones al dirigir el modo en que su cuerpo engrana activamente en el mundo circundante -"lo que él está haciendo: leyendo, danzando, corriendo apresuradamente", etc.), esa reproducción mental puede ir hasta el punto de despertar en nosotros los movimientos correspon-

16 "Es muss gezeigt werden, wie die sinnliche Ähnlichkeit des Körpers 'dort' mit dem Körper, der als mein Leibkörper die Sinnesschichte der Beseelung, zunächst der spezifischen Leiblichkeit [...] trägt und Nullobjekt der 'un inm herum orientierten' erscheinende Welt ist, der Auffassung jenes Körpers den Sinn 'mitdaseiender Leib als Nullglied einer primordinal konstituierten eigenheitlichen Welt mit dem zugehörigen Ich und Bewusstseinsleben' zuwachsen lässt, aber das alles als mitseiend 'appräsentiert', in einer leeren Vergegenwärtigung, die nicht den Charakter der wirklichen Gegenwärtigung, also der Ursprünglichkeit der wirklich primordinalen Gegebenheit annehmen kann". Hua, XV, p. 14. 
dientes. ¿Quién no se ha sorprendido inclinando su cuerpo con el corredor que describe una curva cerrada, pataleando con el futbolista que va realizar un remate, o sea, quién no se ha observado reproduciendo el comportamiento del otro por emparejamiento del otro consigo mismo y aprehendiendo, por causa de este emparejamiento, un objeto del mundo con el sentido -"otro sujeto psicosomático (como yo)"?

Una teoría naturalista de la conciencia debería poder encontrar los centros cerebrales responsables de este fenómeno de reproducción mimética, que, en ciertos casos, puede incluso desencadenar fenómenos motores. Ellos no preocupan a Husserl y tampoco nos debían ocupar aquí. Lo esencial es que este fenómeno general, que designamos como "reproducción mimética", intentando recuperar algunas de las intuiciones centrales de Husserl, implica los siguientes momentos constitutivos:

a) Percepción -o sea, conciencia originariamente donadora de corporalidad (el cuerpo, Körper);

b) Presentificación -transposición analógica en el emparejamiento, o sea, "vaciamiento" sobre la percepción de la corporalidad ajena de la constitución introceptiva de "mí" cuerpo propio (el soma, Leib), bajo la forma de una conciencia no directamente donadora (Wahrnehmung, Gegenwärtigung), sino de una "presentificación" (Vergengenwärtigung) de la senciencia externa, del comando del cuerpo, de las emociones, etc.;

c) Reproducción -interpretación comportamental (bajo la forma de un cuasi-consumar) de la relación del cuerpo ajeno con su mundo circundante, por la remisión para un polo de intenciones y de vivencias subjetivas (la psique, Seele, en cuanto ego ajeno, sujeto de actos y de pasividades), reproducido según el modo "como si yo estuviese allí".

En la intropatía, en la Einfühlung, aquí reinterpretada como reproducción mimética, estos niveles que acabamos de distinguir están escalonados según la relación general de fundación. Por un lado, ninguno de los estratos superiores de sentido es posible sin los estratos inferiores, o sea, la supresión de cualquiera implica la supresión de los estratos fundados, sin que, inversamente, los estratos fundantes carezcan de los otros para hacerse efectivos. Una psique sin soma y un soma sin cuerpo son, por eso, imposibles: una psique sólo se puede realizar somatizada y un soma sólo se puede realizar corporalizado; inversamente, un cuerpo puede ser dado sin soma (se trata del estrato de sentido de 
la "naturalidad"), y un soma puede ser dado sin una vida psíquica superior (se trata del estrato de la "animalidad"). Por otro lado, la aprehensión no está dirigida hacia los estratos inferiores, sino hacia el más alto estrato, el cual reconfigura y impregna los estratos inferiores. Así, no se aprehende primero un cuerpo, después, por inferencia, un soma y, al final, por interpretación, una psique -se aprehende una vida psíquica, pura y simplemente, y la aprehensión de la vida psíquica impregna la aprehensión del soma y la aprehensión del soma impregna la aprehensión del cuerpo, de tal modo que el resultado es la apresentación de una vida psicosomática en su integridad.

Dos cosas son importantes en este contexto. La primera es el modo de visibilidad de otro sujeto. Efectivamente, se da sólo la dimensión corpórea, correlato de la simple percepción. Como Husserl describe, las dimensiones fundadas, que son, no obstante, el centro temático de la aprehensión de un alter-ego, son objeto de una apresentación (Appräsentation) esto es, de una cuasipresentación (Gegenwärtigung), de una cuasi-patencia que jamás puede, sin embargo, transformarse en una aprehensión directa. En efecto, la aprehensión directa de un soma y de una vida psíquica en él impregnada sería una aprehensión según el sentido "yo propio" y no según el sentido "yo ajeno". La apresentación, y la imposibilidad de transformarla en una presentación, es la garantía de que la distancia entre ego y alter-ego es insuperable. Pero -y ésta es la segunda observación- esto no hace de la aprehensión de otro sujeto un proceso inferencial. De hecho, si atendemos a los datos fenomenológicos de la aprehensión intersubjetiva, esto es, a su tenor inmanente de sentido, la patencia de otro sujeto no es inferida a partir del cuerpo físico, sino, al revés, es la aprehensión de una vida psíquica la que dirige la aprehensión del soma y ésta la que dirige la aprehensión del cuerpo. El movimiento es exactamente el inverso - "vemos" inmediatamente a otro ser humano en su unidad, activa y pasiva, con su mundo circundante $y$, porque lo vemos, vemos también su soma $y$, en el límite, el puro cuerpo. Los estratos fundados, que un análisis fenomenológico puede poner al descubierto, no son el resultado de ningún razonamiento inferencial. No hay ahí ningún raciocinio, o sea, ninguna aprehensión activa, categorialmente articulada, de construcción judicativa de nuevas camadas de sentido sobre las anteriores, sino un proceso de génesis pasiva que produce la patencia inmediata (aunque no directa) de su "resultado" -otro sujeto delante de mí. En verdad, es preciso un proceso enteramente artificial de abstracción para 
amputar esta aprehensión inmediata de otro sujeto de su carga de sentido psíquica y somática, para acabar viendo en él un "puro y simple cuerpo". Como es evidente, la aprehensión de otro sujeto no "comienza" ahí, en la corporalidad pura, para desenvolverse después inferencialmente, en pasos sucesivos. Si tenemos ahora al alter-ego en una aprehensión inmediata, jamás hemos tenido antes, por eso mismo, un simple cuerpo, a no ser como pre-donación pasiva, ya desde siempre impregnada por los estratos superiores.

\section{El SENTIDO "OTRO SUJETO"}

Regresemos brevemente a las cuestiones iniciales: la de la génesis fenomenológica del sentido alter-ego, la de la motivación que soporta la posición de la alteridad y la tan comentada "objeción del solipsismo". Hagámoslo globalmente, interpretando algunas de las enseñanzas de los análisis fundamentales de Husserl y de Sartre.

La teoría fenomenológica de la génesis no describe ni la aparición factual de una formación de sentido, ni hace un simple retorno a la evidencia donadora. Si el primer sentido de génesis es pre-fenomenológico y tiene que ver con el origen en un sentido empírico y mundano, el segundo sentido, que es ya un tema y una tarea propia de la fenomenología, tiene, sin embargo, que ver con el mero análisis estático de la correlación noético-noemática. Se trata, ahí, de buscar, para una formación de sentido ya dada, la intuición que la rellena y la evidencia correspondiente. Génesis, en el sentido fenomenológico pertinente, es la exhibición del proceso por el cual una apercepción se edifica a partir de apercepciones pre-dadas, en una historicidad puramente ideal, que progresa de una institución primitiva de sentido (una Urstiftung, en la designación de Husserl) a otra y que, en el límite, apenas presupone la continuidad subjetiva de la vida, a partir de la cual todas las apercepciones deben ser exhibidas como formaciones de sentido y validaciones formándose en ella. El punto de partida no es la conciencia (Bewusstsein), en su polaridad noético-noemática estática, sino la vida 
(Leben) en el proceso de autoconstitución de su temporalidad inmanente y de su historicidad propia, como institución progresiva de sentido ${ }^{17}$.

Una cosa puede ser dicha en la estela de algunas ideas centrales de Husserl: un ego humano sólo tiene sentido en plural, o sea, la posición de una pluralidad de sujetos es una condición sin la cual no se hace efectiva una experiencia de mundo; y la creencia en la cual se hace efectiva la posición del mundo como horizonte de experiencia no es, por consiguiente, apenas posición de simples cosas, sino también -y al mismo tiempo- de otros ego, bajo la forma de sentido alter. Esto significa que la posición de un complejo de existencias cósicas es, por esencia, su posición no sólo "para mí", sino "para cualquiera" (für jedermann). Que la experiencia de cosas (o sea, de una objectualidad en el estrato de sentido de la "simple naturaleza") presuponga ya la pluralidad de los ego, es un resultado firme de los análisis constitutivos de la quinta meditación cartesiana. La objetividad sólo es según la forma de la intersubjetividad. Esta posición de la pluralidad de los ego, inscrita en la propia Weltdoxa, es, por otro lado, la forma de efectivación mundana de una pluralidad trascendental de sujetos monádicos, por cuya concordancia entre las vidas constitutivas despunta el mundo como horizonte común de experiencia. Dos cosas son, aquí, importantes: primero, la forma intersubjetiva de la experiencia está presupuesta en la posición de una realidad mundana y de un ego mundano que se refiere a ella, y está presupuesta según la forma de la intersubjetividad abierta (offene Intersubjektivität), o sea, según la forma del para-alguien, que es otro ego posible; segundo, la descripción del modo en que se hace efectiva la aprehensión de otro ego en el horizonte de la experiencia mundana (conteniendo ya una intersubjetividad abierta) no es la génesis del sentido alter-ego, sino la forma de su cumplimiento intuitivo, según la forma apresentativa (no-presentativa) que le es peculiar. Así, describir el proceso de la aprehensión por empatía de otro ego en su existencia presente no es describir la génesis del sentido alter,

17 "Diese 'Geschichte' des Bewusstseins (die Geschichte aller möglichen Apperzeptionen) betrifft nicht die Aufweisung faktischer Genesis für faktische Apperzeptionen [...], vielmehr jeder Gestalt von Apperzeptionen ist eine Wesensgestalt und hat ihre Genesis nach Wesensgesetzen, und somit liegt in der Idee solcher Apperzeption beschlossen, dass sie einer 'genetischen Analyse' zu unterziehen ist. [...] Die Theorie des Bewusstseins ist also geradezu Theorie der Apperzeptionen; der Bewusstseinsstrom ist ein Strom einer beständigen Genesis, nicht ein blosses Nacheinander, sondern Auseinander, ein Werden nach Gesetzen notwendiger Folge, in dem aus Urapperzeptionen oder aus apperzeptiven Intentionen primitiver Art konkrete Apperzeptionen von verschiedener Typik erwachsen [...] In gewisser Weise scheidet sich also 'erklärende Phänomenologie' als Phänomenologie der gesetzmässigen Genesis und 'beschreibende' Phänomenologie als [...] 'statische' Phänomenologie". Hua XI, pp. 339s. 
sino circunscribir el modo en que ese sentido puede ser cumplido por una evidencia correspondiente. Estamos, en este nivel, todavía en el plano de un análisis estático o de una "fenomenología descriptiva", no genética.

¿De dónde el sentido alter, entonces? Los análisis de Husserl, en las Meditaciones cartesianas, sobre la dicha esfera de "primordialidad" -la "mía-propia" (Mir-eigene)-, nos permiten lanzar una mirada penetrante sobre esta cuestión. Pero es preciso comprender bien lo que Husserl intenta hacer ahí. La esfera primordial, que se define como experiencia sin el sentido alter-ego, representa simplemente una abstracción metodológica. La reducción a la esfera de propiedad, que Husserl realiza en el $\S 44$, no es la exhibición de una forma primitiva de la experiencia, sino la amputación de todos los sentidos de la experiencia que tengan que ver directamente o que supongan implícitamente el sentido alter-ego, para hacer visibles, en negativo, las implicaciones del sentido "otro sujeto" en la experiencia mundana. La experiencia no comienza ahí, obviamente, en la esfera de propiedad de un ego privado de alter-ego y de todo lo que está pendiente de este último. Se trata, mediante esta reducción artificial, de medir en toda su amplitud las formaciones de sentido que, en la experiencia del mundo, suponen ya la posición de otro sujeto, sea bajo la forma de una intersubjetividad abierta (für jedermann), sea bajo la forma de una experiencia concreta de otro sujeto en la empatía. Por tanto, el orden del progreso de los análisis de Husserl no es un verdadero orden de derivación de las apercepciones en causa. La experiencia no comienza con un ego solipsista que, en pasos sucesivos, constituye otro sujeto y un mundo objetivo ahí transcurriendo. El orden inverso es el correcto y todas las reflexiones de Husserl, en la quinta meditación, deben, por tanto, ser regresivamente reconstituidas a partir de lo que en ellas es resultado, yendo de la experiencia del mundo en su plena concreción hasta sus ingredientes parciales, que sólo una abstracción metódica permite aislar.

El § 49 de las Meditaciones cartesianas indica las etapas del análisis:

1. Esfera de pertenencia, o la experiencia según el sentido exclusivo del yo propio y del para mí -"mundo primordial";

2. Yo ajeno -constitución de un mundo natural, a partir de la diferencia entre mi aparición y el mundo "objetivo", idéntico para cualquier otro (en cuanto sistema de apariciones subjetivas armonizadas); 
3. Comunidad de los ego y el "nosotros" comunitario, como sistema congruente de experiencias de un mundo común, subyacente a los flujos de apariciones subjetivas;

4. Formas superiores de comunidad en los actos específicos de comunicación (actos de un "yo" dirigido apresentativamente a un "tú"), productores de la sociabilidad -constitución de una comunidad de "segundo grado", en cuanto comunidad socializada en las formas objetivas de la cultura ( $§ 58$ ).

Casi incidentalmente, Husserl expresa tres tesis esenciales: (1) que la figura en sí primera del otro es el otro yo (y que sólo eso hace posible la alteridad "segunda" de una Naturaleza trascendente) ${ }^{18}$; (2) que la constitución de un mundo común implica un proceso de armonización de las vidas monádicas, constituyente de una comunidad ${ }^{19}$; (3) que la comunidad intersubjetiva se constituye en actos sociales en la forma de la comunicación ${ }^{20}$. O sea, por su orden intrínseco (y no por el orden progresivo, a partir de la figura abstracta de un "mundo primordial") -que la primera apercepción es la de la trascendencia de un alter-ego (y no la de un mundo objetivo cósico); que la conciencia empática de otro ego se desenvuelve como interacción comunicativa en actos de socialización, productores de objetividades intersubjetivas y de una esfera de cultura (como sedimento de las realizaciones comunicativas); que la interacción comunicativa es el proceso de armonización de las vidas monádicas o de la congruencia multipolar de los flujos de experiencia (apuntando a la idea-límite de un consenso comunicativo); que la congruencia de los flujos de experiencia hace posible un Mundo y, en particular, una Naturaleza, como conciencia de un fondo perceptivo invariable por debajo de la variación de las formaciones de la cultura (y que la propia idea de una Naturaleza "objetiva y exacta", en el sentido de las ciencias de la naturaleza, será una formación cultural de tipo determinado).

Así, reinterpretado el movimiento de la quinta meditación, la tesis fundamental que se extrae de las largas reflexiones de Husserl sobre el tópico de la intersubjetividad es la de que la forma en sí primera de la vida de experiencia

\footnotetext{
18 "Also das an sich erste Fremde (das erste 'Nicht-Ich") ist das andere Ich". Hua I, p. 109.

19 "Die transzendentale Intersubjektivität hat durch diese Vergemeinschaftung eine intersubjektive Eigenheitssphäre, in der sie die objektive Welt intersubjektiv konstituiert. [...] Danach gehört zur Konstitution der objektiven Welt wesensmässig eine 'Harmonie' der Monaden". Hua I, p. 110.

20 "[Es gibt] spezifisch ichlich-personalen [Akten], die den Charakter von Ich-Du-Akten, von sozialen Akten haben, durch welche alle menschliche personale Kommunikation hergestellt wird". Hua I, p. 135.
} 
es la apercepción de un alter-ego, y que son los procesos empáticos y comunicativos los que conducen a la edificación de una comunidad. El ego solo en relaciones comunicativas (reales o posibles) puede tener delante de sí algo como un alter-ego. Y este "tener-delante" es, más fundamentalmente, un "estarcon", en la unidad de un mundo socializado constituyéndose a lo largo de esas relaciones comunicativas. La forma fundamental de la conciencia de otro sujeto consiste en estos procesos comunicativos y empáticos de armonización de los flujos de experiencia respectivos, por los cuales despunta la forma comunitaria de un "nosotros" socializado y la referencia a un mundo común (cultural y natural). En verdad, sin los procesos de armonización comunicativa de los flujos de experiencia, sin, por lo tanto, la conexión de la auto-aparición con la aparición de un alter-ego y la conciencia recíproca de una comunidad, que ahí se edifica, no hay, para el ego, ninguna cosa así como una efectiva experiencia de mundo: la experiencia de un mundo es obra de un ego que desde siempre fue ya conciencia de una comunidad (real o posible) de sujetos en conciencia recíproca de la armonía de sus flujos de experiencia -tal es la última lección que deriva de la lectura que hacemos del movimiento global de la quinta meditación. Desde este punto de vista, es bien cierto que, para Husserl, la forma de una comunidad intermonádica es el verdadero punto de partida de una filosofía trascendentalconstitutiva, como lo ha mostrado Dan Zahavi ${ }^{21}$, y que sólo mediante una abstracción unilateral (de la que el propio Husserl fue responsable por ciertas presentaciones de la Fenomenología, destacadamente en lo que se refiere a la "vía cartesiana") se puede hablar de un ego encapsulado en una conciencia apodíctica de sí mismo que convierte el sentido alter-ego en un problema irresoluble.

Esta comprensión de la intersubjetividad comunitaria como forma en sí primera de una efectiva experiencia de un mundo común, cultural y natural, entra, ahora, en un contraste remarcable con los análisis sartreanos de la intersubjetividad. Guiado por su idea central de que la conciencia de otro sujeto es el lugar donde despunta una libertad que me hace nacer como yo-objeto, y que ese ser objectual no es recuperable sino mediante una imposible absorción de la libertad del otro -guiado por la idea de que el fondo siempre presente de las

${ }^{21}$ Cfr. Dan Zahavi, Husserl und die transzendentale Intersubjektivität. Eine Antwort auf die spachpragmatische Kritik, Dordrecht, Kluwer Academic Publishers, 1995. 
relaciones intersubjetivas es el conflicto entre las libertades, que se aniquilan mutuamente en el movimiento circular del ver y del ser-visto-, la forma de la comunidad intersubjetiva se vuelve, para Sartre, un problema que sólo puede ser efectivamente resuelto mediante la alteración de los conceptos fundamentales de su análisis. Estos están especialmente dirigidos a una minuciosa descripción del modo en el que la conciencia de un alter-ego es un verse en el ver del otro, o sea, una conciencia del modo en el que somos objeto para él, y no del modo en el que devenimos sujetos, con él, en una comunidad intersubjetiva. Lo esencial de sus análisis de la intersubjetividad está dado de un modo claro en la estructura del fenómeno de la vergüenza (honte):

"Yo tengo vergüenza de mí mismo", la vergüenza supone un yo-objeto para el otro, pero también una ipseidad que tiene vergüenza y que es imperfectamente expresada por el "yo" de la fórmula. Así, la vergüenza es aprehensión unitaria de tres dimensiones: "Yo tengo vergüenza de mí ante el otro". ${ }^{22}$

El núcleo esencial de la conciencia de otro sujeto es, así, el desprendimiento de sí, el mal-estar, la alienación y la caída en una forma de ser que, al mismo tiempo, no es el para-sí, sino que le aparece como irrecusable. Esta forma de relación consigo mismo en la objetivación y la conciencia de otro sujeto son, para Sartre, una y la misma cosa, de tal modo que, partiendo de este concepto unilateral de la relación intersubjetiva, las formas de la socialización y de la comunidad superior del "nosotros" se vuelven, en el fondo, incomprensibles a partir de sus análisis. Este pensamiento fundamental es retomado en muchos lugares. Un último ejemplo:

Así, mi yo-objeto no es conocimiento ni unidad de conocimiento, sino mal-estar, desprendimiento vivido de mi unidad ek-stática del para-sí, límite que no puedo alcanzar y que sin embargo yo soy. Y el otro, a través del cual ese yo me adviene, no es conocimiento ni categoría, sino el hecho de la presencia de una libertad extraña. En efecto, mi desprendimiento de mí y el surgimiento de la libertad del otro constituyen una sola cosa; sólo puedo sentirlos y vivirlos juntos; no puedo ni siquiera intentar concebirlos el uno sin el otro. ${ }^{23}$

22 "En effet dans la structure qu'exprime le 'j'ai honte de moi', la honte suppose un moi-objet pour l'autre más aussi une ipséité qui a honte et qu'exprime imparfaitement le 'Je' de la formule. Ainsi la honte est appréhension unitaire de trois dimensions: 'J'ai honte de moi devant autrui'". L'être et le néant, p. 337.

${ }_{23}$ "Ainsi mon moi-objet n'est ni connaissance ni unité de connaissance, mais malaise, arrachement vécu à l'unité ek-statique du pour-soi, limite que je ne puis attendre et que pourtant je suis. Et l'autre, par qui ce moi $m^{\prime}$ arrive, n'est ni connaissance ni catégorie, mais le fait de la présence d'une liberté étrangère. En fait, mon arrachement à moi et le surgissement de la liberté d'autrui ne font qu'un, je ne puis les ressentir et les vivre qu'ensemble, je ne puis même tenter de les concevoir l'un sans l'autre". Ibidem, p. 322. 
Si en Husserl encontramos un "solipsismo" metodológico destinado a mostrar que el sentido de la subjetividad es la intersubjetividad comunitaria, en la empatía y en la comunicación, autora de la socialidad, en Sartre tenemos, al revés, una afirmación inicial del otro como la insuperable presencia de una libertad que aniquila la mía propia, me deja ver en ella apenas mi propia imagen como objeto, y que para siempre me encierra en una sombría soledad.

Traducido por Francisco Conde Soto 